\title{
Lymphadenopathy After BCG Vaccination in a Child with Chronic Granulomatous Disease
}

\author{
Ana Paula Vieira, M.D.,* Júlia Vasconcelos, M.D.,† José Carlos Fernandes, M.D.,* Henedina \\ Antunes, M.D., $\div$ A. Sousa Basto, M.D.,* Cristiana Macedo, M.D.,* Afsana Zaman, M.D.,* \\ Eugénia Santos, M.D. $\dagger$ J. Castro Melo, M.D., $\dagger$ and Dirk Roos, M.D. \\ Departments of *Dermatology and $\ddagger$ Pediatrics, S. Marcos Hospital, Braga, Portugal; $\dagger$ Department of Immunology, S. \\ António Hospital, Porto, Portugal; and §Sanquin Research, Landsteiner Laboratory, Academic Medical Center, \\ University of Amsterdam, Amsterdam, The Netherlands
}

\begin{abstract}
We report a 15-month-old boy who developed an ulcer in the left axillary fold following bacillus Calmette-Guérin vaccination. Subsequent immunologic and genetic studies led to the diagnosis of chronic granulomatous disease. His mother had "lupus-like" lesions, described in some carriers of this disease, that were thus related to her son's diagnosis. Although in healthy subjects this vaccination is usually harmless, in instances of impaired immunity it may cause adverse reactions. When a vaccine-related complication occurs, an underlying immunodeficiency should be sought.
\end{abstract}

Bacillus Calmette-Guérin (BCG) vaccination is usually harmless, however, it can cause specific complications resulting from the interaction of the host with the live bacillus. The frequency of these complications is not well known, but the incidence is certainly very low if we consider the large number of vaccines that are administered yearly (1). Benign regional adenitis is the most common complication, especially in the younger age group. Some patients may develop scrofuloderma, which can persist for $6-12$ months $(1,2)$. In cases of impaired immunity, vaccination can cause disseminated BCG infection, which may be lethal $(3,4)$.

Chronic granulomatous disease (CGD) is a rare primary immunodeficiency in which a deficiency of the nicotinamide adenine dinucleotide phosphate (NADPH) oxidase enzyme complex, which generates microbicidal reactive oxygen species in phagocytes, renders these cells inefficient at killing the ingested microorganisms.
As a consequence, recurrent infections with poor response to therapy are common, with abscess formation in the skin, soft tissue, lungs, and liver and suppurative lymphadenopathy in the cervical lymph nodes $(5,6)$. Excessive formation of granulomas in all tissues is also common (7).

We report a 15-month-old boy who developed axillary lymphadenopathy after BCG vaccination. After subsequent immunologic function and genetic investigations, a diagnosis of CGD was made. The child's mother had "lupus-like" lesions on her face and hands, as previously described in carriers of CGD.

\section{CASE REPORT}

An apparently healthy 15-month-old boy with a good nutritional status and growth in the 25th percentile presented to the dermatology service in May 2000 with

Address correspondence to Ana Paula Vieira, M.D., Serviço de Dermatologia, Hospital de S. Marcos, Apartado 2242, 4701-965 Braga, Portugal, or e-mail: dermato@hsmbraga.min-saude.pt. 


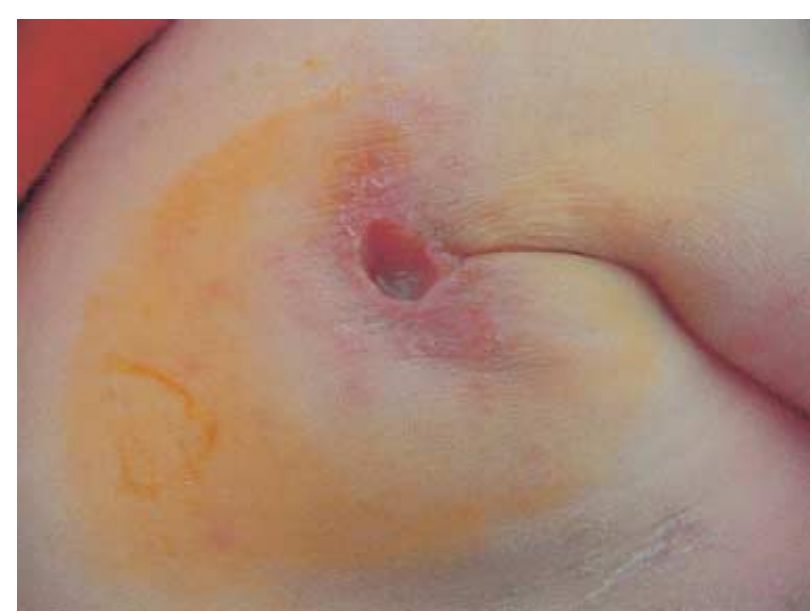

Figure 1. Ulcer in the left axillary fold.

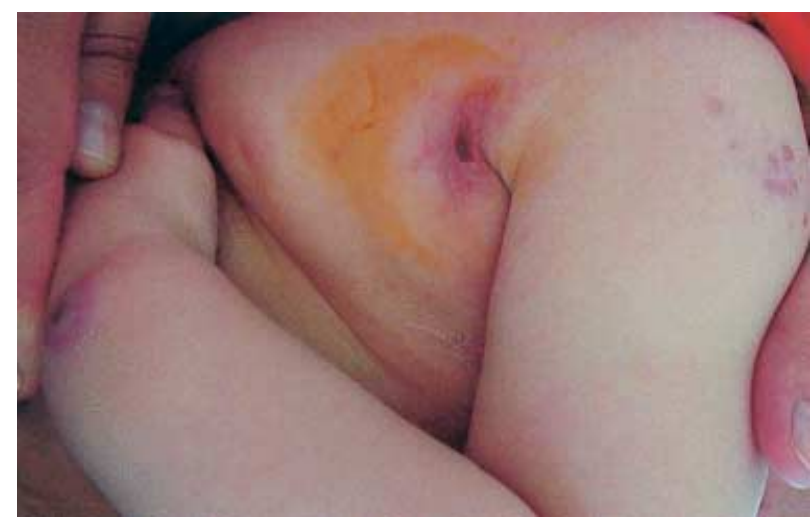

Figure 2. Ulcer in the left axillary fold and nodular lesion on the wrist.

a deep ulcer in the left axillary fold (Figs. 1 and 2) that had developed over the previous year. This lesion had evolved from lymphadenopathy that had appeared 3 months after BCG vaccination. The child also had a soft, erythematous, nodular lesion on the left wrist of 3months duration (Fig. 2). Except for these lesions, the physical examination was normal, with no evidence of other lymphadenopathy or hepatosplenomegaly.

Previously the child had been treated for 7 months with isoniazid, which did not improve his ulcer or prevent the appearance of an additional lesion on the wrist. His past medical history included separation of the umbilical cord at 15 days after birth, several upper respiratory infections, and a urinary tract infection that subsided quickly with antibiotics. He had a routine vaccination program (diphtheria, pertussis, and tetanus [DPT] vaccine and anti-Haemophilus vaccine at 2,4 , and 6 months of age, measles-mumps-Rubella [MMR] at 15 months, and a booster of Haemophilus vaccine at 18 months) without any complications.

His family history was of significance in that the child's mother, age 24 years, has had relapsing erythematous, maculopapular lesions on her hands since childhood. More recently, similar lesions also appeared on her face. She had been followed at the dermatology department for suspicion of lupus erythematosus, although this diagnosis was never confirmed, despite several tests for autoantibodies and studies of skin biopsy specimens.

Blood studies of the child found a hemoglobin of 9.3 $\mathrm{g} / \mathrm{dl}$, hematocrit $28.9 \%$, anisocytosis, anisochromasia, and poikilocytosis. Results within the normal range were obtained for the number of leukocytes (and for neutrophils), for tests of hepatic and renal function, electrophoresis of proteins, and $\alpha_{1}$-antitrypsin. Erythrocyte sedimentation rate and C-reactive protein were elevated (38 $\mathrm{mm}$ and $26.2 \mathrm{~m} / \mathrm{L}$, respectively). Serum levels of $\operatorname{IgA}$ and $\mathrm{IgG}$ were elevated $(156 \mathrm{mg} / \mathrm{dl}$ and $1710 \mathrm{mg} / \mathrm{dl}$, respectively), and antinuclear antibodies were negative. The cutaneous Mantoux test was negative $(5 \mathrm{~mm})$. A chest radiograph and renal and pelvic ultrasound scans did not detect any anomaly. Histopathology of the axillary and wrist lesions showed a chronic granulomatous process with giant multinucleated cells. No alcohol-acid resistant bacilli were detected in the biopsy specimens nor in the exudate of the lesion.

In view of these results, the decision was made to add rifampicin to the treatment with isoniazid. After 3 months of this double antituberculosis therapy, there was complete regression of the wrist lesion and marked improvement of the axillary ulcer, which was reduced to a small exudative lesion. Medication was discontinued due to diarrhea that was difficult to control. Two months later a new nodular lesion, similar to the one on the wrist, appeared close to the left labial commissure (Fig. 3).

A detailed immunologic study was performed to confirm the suspicion of a primary immunodeficiency. Immunophenotyping of the B, T, and natural killer (NK) cells did not reveal any significant abnormalities other than evidence of strong T-cell activation (increased expression of HLA-DR and CD45RO), which was ascribed to recent infections. Expression of adhesion molecules (CD18/CD11) and myeloperoxidase on the leukocytes were normal. Functional studies of phagocytic cells showed a profound reduction in the oxidative burst of the granulocytes and monocytes (Table 1) when analyzed by flow cytometry as the fluorescence emitted by oxidation of dihydrorhodamine (Bursttest, Orpegen Pharma, Heidelberg, Germany) upon stimulation of the cells with Escherichia coli or phorbol myristate acetate (PMA). These results are fully compatible with 


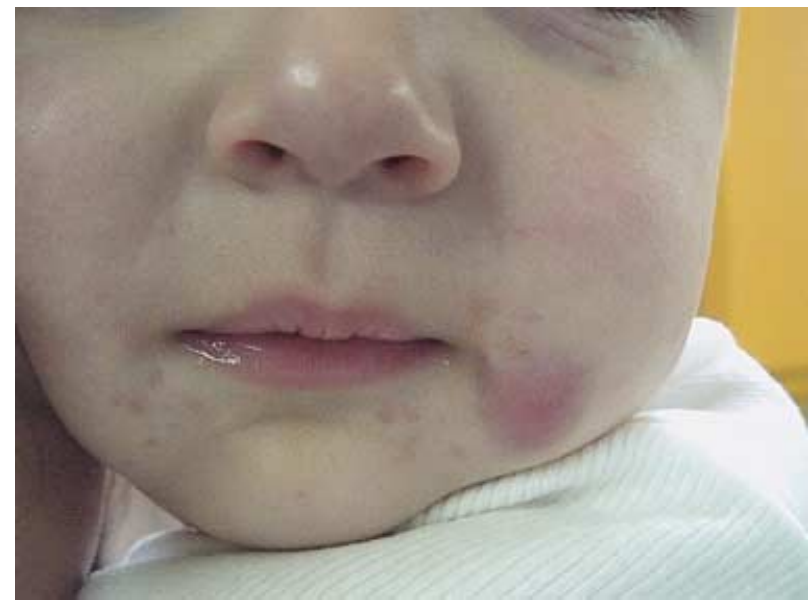

Figure 3. Nodular lesion on the face.

TABLE 1. Results of the Oxidative Burst of the Granulocytes and Monocytes of the Patient Activated with PMA or E. coli

\begin{tabular}{|c|c|c|}
\hline Activation & $\begin{array}{l}\text { Percent positive cells } \\
\text { (patient/50 controls) }\end{array}$ & $\begin{array}{l}\text { Median fluorescence intensity } \\
\text { of the positive cells (channel) } \\
\text { (patient } / 50 \text { controls) }\end{array}$ \\
\hline PMA & Granulocytes: 12/96-100 & Granulocytes: $0.930 / 19.6-81.15$ \\
\hline \multirow[t]{2}{*}{ E. coli } & Granulocytes: 79/94-100 & Granulocytes: $1.330 / 6.59-31.53$ \\
\hline & Monocytes: 22/34-96 & Monocytes: 0.800/3.07-11.09 \\
\hline
\end{tabular}

abnormalities usually seen in CGD. The expression of the NADPH oxidase protein gp91 $1^{\text {phox }}$ by the phagocytic cells studied by flow cytometry with mAb 7D5 was undetectable, indicating that the patient probably had an $\mathrm{X}$-linked form of CGD.

The study of the oxidative burst of the phagocytic cells of the patient's mother (after stimulation with $E$. coli or PMA) clearly discriminated two granulocyte populations, with $60 \%$ of the cells showing a markedly reduced oxidative burst, while the remaining $40 \%$ had normal fluorescence. In concordance with these results, the expression of gp91 $91^{\text {phox }}$ was undetectable in $57 \%$ of the mother's granulocytes, but showed normal levels in the remaining cells. These results confirm that the mother is a carrier of X-linked CGD and hence indicate that the patient's defect is inherited.

The molecular characterization of the genetic defect, by mutation analysis of DNA, revealed a mutation in the $\mathrm{X}$-CGD gene $C Y B B$. This mutation consists of an inserted $\mathrm{G}$ after the G-6 and leads to a frame shift and to a stop codon in codon 8 . The same mutation was detected in heterozygous form in the mother.

After a 1-month suspension, antituberculosis therapy with isoniazid and rifampicin was resumed for 12 months, with good results. At this time, a prophylactic treatment with trimethoprim-sulfamethoxazole $(4 \mathrm{mg} / \mathrm{kg} /$ day, twice a day) and itraconazole $(5 \mathrm{mg} / \mathrm{kg} /$ day, twice a day) was also started and has been maintained up to the present time with no secondary effects. Presently the child remains without infection or any other complication.

\section{DISCUSSION}

Vaccination with the attenuated strain of Mycobacterium bovis bacilli Calmette-Guérin is well tolerated and adverse reactions are rare, although it is the one of the most widely used vaccines in the world. When reactions do occur, specific complications of the vaccination usually consist of benign lymphoglandular and skin reactions that mimic cutaneous responses to "natural" mycobacterial infection $(1,2)$. Occasionally, in children with impaired immunity, disseminated BCG infection may occur, and sometimes is lethal. Most of these children have combined immunodeficiency and some have CGD $(3,4)$.

Initially our patient's suppurative lymphadenitis was interpreted as a specific complication of the BCG vaccine and, as recommended by several protocols, treatment with isoniazid was started (8). Failure of this treatment regime, denoted by the associated emergence of cutaneous granulomas at other sites, led us to investigate a possible immunodeficiency, and as a result, the diagnosis of CGD was made.

Chronic granulomatous disease was definitively recognized as a disease of the phagocytes in the 1960s, when it was demonstrated that neutrophils of these patients have a specific deficiency in the intracellular killing of bacteria. This deficiency was traced to a defect in the oxidative metabolism of phagocytes, manifested as an absence of the "respiratory burst" that occurs during phagocytosis $(9,10)$. The oxidative activity of phagocytes is catalyzed by NADPH oxidase, which performs the monovalent reduction of molecular oxygen $\left(\mathrm{O}_{2}\right)$ to superoxide anions $\left(\mathrm{O}_{2}^{-}\right)$. This free radical is in turn converted (spontaneously or catalyzed by superoxide dismutase) to hydrogen peroxide $\left(\mathrm{H}_{2} \mathrm{O}_{2}\right)$, which can be used by the neutrophil enzyme myeloperoxidase (MPO) for conversion into microbicidal intermediates that cause the destruction of the phagocytized microorganisms $(6,9)$. NADPH oxidase is a multicomponent enzyme, with membrane-bound components, the $91 \mathrm{kDa}$ glycoprotein $\mathrm{gp} 91^{\text {phox }}$, and the $22 \mathrm{kDa}$ protein $\mathrm{p} 22^{\text {phox }}$, which functionally interact with cytoplasmic components, the $47 \mathrm{kDa}$ protein $\mathrm{p} 47^{\text {phox }}$, and the $67 \mathrm{kDa}$ protein $\mathrm{p} 67^{\text {phox }}$ $(9,11)$. Mutations in the genes of any of these four proteins can cause CGD. Since $C Y B B$, the gene that 
encodes $\mathrm{gp} 91^{\text {phox }}$, is located on the $\mathrm{X}$ chromosome, mutations in $C Y B B$ cause the $\mathrm{X}$-linked form of CGD.

Chronic granulomatous disease is a rare disease, with an estimated incidence of 1 in 250,000, and has a marked male predominance due to the most common mode of genetic inheritance (11). Manifestations of the disease are usually seen before the age of 2 years as recurrent infections on the epithelial surfaces that are in direct contact with the environment, such as the skin, lungs, and gastrointestinal tract (12). The most common presenting feature is lymphadenitis, particularly of the cervical nodes, followed by cutaneous abscesses, pneumonia, hepatomegaly, and diarrhea. Hepatic abscess, osteomyelitis, and sepsis may also occur (12). In addition, there is an excessive formation of granulomas in all tissues (7). The main cutaneous manifestations include abscesses, pyoderma with local lymphadenopathy, and dermatitis in periorificial areas $(5,6)$. In the literature there are descriptions of oral and perioral ulcerations, chronic gingivitis, scalp folliculitis, chronic suppurative paronychia, and poor healing of surgical wounds (6).

The microorganisms responsible for most of the infections in CGD are catalase-positive bacteria such as Staphylococcus aureus and gram-negative Enterobacteriaceae, including Salmonella, Klebsiella, Aerobacter, Serratia, and some species of Pseudomonas, particularly Burkholderia cepacia. The fungus most commonly implicated in CGD is Aspergillus, predominantly A. fumigatus $(6,7)$. Catalase-negative bacteria like Streptococcus rarely cause problems, since the small amounts of hydrogen peroxide that these bacteria produce are not degraded by catalase and are therefore available within the phagocytic vacuoles for the production of microbicidal intermediates (9). Less common than infections are cutaneous granulomas, which are frequently nodular and necrotic; these can appear at the inoculation site $(6,9,11)$. Mycobacterial disease is uncommon in patients with CGD, but draining skin lesions and lymphadenopathy can occur at the site of BCG inoculation. Focal or miliary pulmonary disease may arise as a result of infection with nontuberculous mycobacteria, and may be followed by pulmonary fibrosis (7).

Children with CGD usually have short stature and low weight. The end of the first decade of life represents a turning point for these patients, as thereafter the infections are usually less frequent and less severe $(11,12)$. In laboratory tests, one frequently sees hypergammaglobulinemia, anemia, and an increased erythrocyte sedimentation rate, probably as a reflection of an ongoing subclinical inflammation status $(11,12)$.

The diagnosis of CGD is based on laboratory demonstration of the inability of phagocytes from affected individuals to produce a normal respiratory burst. For that purpose, the nitroblue tetrazolium (NBT) test was the classic test, but now oxidation of dihydrorhodamine to rhodamine, analyzed by flow cytometry, is the preferred method to quantitatively assess the NADPH oxidase activity of phagocytes. In this test, the intensity of rhodamine per cell is measured, as well as the fraction of positive and negative cells. This last property allows detection of the carrier status in instances of X-linked $\mathrm{CGD}$, because carriers of $\mathrm{X}$-linked diseases possess a mosaic of normal and deficient cells due to the random inactivation of one X chromosome in female cells. Further confirmation and characterization of the defect at the molecular level can be obtained by DNA analysis and direct sequencing of the relevant gene $(11,12)$.

About two-thirds of the cases of CGD are caused by defects in $\mathrm{gp} 91^{\text {phox }}$ encoded by the $C Y B B$ gene located on the $\mathrm{X}$ chromosome (Xp21.1), and since the expression of this form is recessive, the disease as a whole is more common in males. The genes for the other subunits of the enzyme are located on autosomal chromosomes, and therefore the remaining cases, comprising predominantly defects in $\mathrm{p} 47^{\text {phox }}$, are inherited as autosomal recessive traits $(6,7,9,11)$. In X-linked CGD (XL-CGD), several types of mutations have been identified, the most common being a frame shift due to an insertion of an adenosine after position 754 , where six consecutive adenosines may predispose to recombination errors (9). In our patient, the mutation in the X-CGD gene $C Y B B$ was a frame shift due to an insertion of guanine after G-6, leading to a stop codon in codon 8 .

Cell-mediated immunity in CGD is usually not affected, even though the macrophages and granulocytes of these patients have a reduced bactericidal capacity (13). As described in this case report, there were appropriate responses to MMR, DPT, and polio vaccinations, indicating that cellular immunity was preserved.

Resistance to Mycobacterium tuberculosis is achieved through the cooperation between helper $\mathrm{T}$ lymphocytes and macrophages (delayed hypersensitivity type of cellular immunity): stimulation, such as that produced by mycobacterial infection, causes macrophages to secrete tumor necrosis factor (TNF)- $\alpha$, interleukin (IL)-12, and other factors that promote differentiation and activation of CD4 Th1 cells to secrete interferon (IFN) $-\gamma$ and other macrophage-activating factors. Macrophages, in response, secrete TNF- $\alpha$ and activate mycobactericidal mechanisms such as nitric oxide production (4).

The pathogenesis of disseminated or protracted local tuberculous infection still remains to be clarified and $M$. tuberculosis has not been described as a major pathogen in patients with CGD $(9,14)$. However, taking into account that the BCG organisms are catalase positive 
and are not killed by CGD phagocytes, it is possible that even in the apparent absence of impairment of the cellular immunity, the macrophage defect alone may predispose patients with CGD to develop BCG infections (13). Defense mechanisms of the phagocytic cells against $M$. tuberculosis are still unclear, but our findings suggest that the respiratory burst can play a role $(7,14)$.

The diagnosis of BCG infection can only be confirmed by identifying the bacilli through appropriate staining, culture, or DNA detection. The histopathologic similarity of granulomas that occur in CGD and BCG renders the diagnosis of the tuberculous etiology difficult in patients with CGD. However, inability to identify the bacilli through culture doesn't exclude tuberculous etiology, and antibacterial treatment is recommended $(8,13)$. Thus, following this recommendation, our patient was successfully treated with isoniazid and rifampin.

Although the evidence obtained was suggestive, it does not allow us to conclude that the adverse reaction to the BCG vaccination in our patient was a consequence of his CGD. However, like other authors, we believe that an unhealed immunization site or the development of regional lymphadenopathy after a BCG vaccination should raise the suspicion of CGD (13). Accordingly, a child who has been diagnosed with CGD or is suspected of having CGD should not be subjected to BCG vaccination (13).

The treatment strategy for CGD consists of aggressive treatment of established infections with antibiotics, and surgical drainage and/or resection of the infectious focus, followed by prophylactic treatment with trimethoprim-sulfamethoxazole or dicloxacillin for infections. Itraconazole can be used for antifungal prophylaxis $(11,12)$. Although not universally used, the prophylactic administration of recombinant IFN- $\gamma$ seems beneficial $(9,11,12)$.

In spite of a retrospective study conducted in 1989 showing a survival rate of $50 \%$ at 10 years of age, the actual prognosis is better now, and most patients survive well into adulthood (12). CGD, being a well-characterized inherited disease, is an ideal candidate to be among the first diseases to benefit from advances in gene therapy $(9,11,12,15)$.

Usually there is no increased susceptibility to infection in female carriers of X-linked CGD, since they have enough phagocytes with functional NADPH oxidase to produce an effective host defense $(6,9,11)$. However, they are predisposed to the development of recurrent stomatitis, erythematous macular, papular, and urticarial skin lesions following light exposure and discoid lupus erythematous (DLE) $(6,9,11,16)$. Nevertheless, some authors suggest a tenuous link between CGD and DLE (16), even though in the majority of cases there were unspecific erythematous rashes, predominantly on photoexposed areas, usually described as "lupus-like," as was the case in our patient's mother. This woman had been followed at the dermatology department over a number of years without any definitive diagnosis until her son's CGD was discovered. Some authors believe that this dermatosis occurring in X-linked CGD carriers should have its own identity, even though it shares characteristics with other conditions, namely DLE (16).

Prenatal diagnosis of CGD can be made by analysis of DNA from cells of the amniotic fluid or chorionic villi sampling obtained during the 10th-12th week of gestation. This strategy is dependent on identification of specific family mutations or on informative polymorphisms. Alternatively, measuring the activity of NADPH oxidase in fetal blood samples during the second trimester can give the diagnosis $(9,11,12)$.

\section{REFERENCES}

1. Tappeiner G, Wolff K. Tuberculosis and other mycobacterial infections. In: Freedberg IM, Eisen AZ, Wolff K, et al, eds. Dermatology in general medicine. New York: McGraw-Hill, 1999.

2. Lotte A, Wasz-Hockert O, Poisson N, Dumitrescu N, Verron M, Couvet E. BCG complications: estimates of the risks among vaccinated subjects and statistical analysis of their characteristics. Adv Tuberc Res 1984;21:107-193.

3. Casanova JL, Jouanguy E, Lamhamedi S, Blanche S, Fischer A. Immunological conditions of children with BCG disseminated infection. Lancet 1995;346:581.

4. Jouanguy E, Altare F, Lamhamedi S, et al. Interferongamma-receptor deficiency in an infant with fatal Babille Calmette-Guérin infection. N Engl J Med 1966;335:19561961.

5. Paller AS. Genetic immunodeficiency diseases. In: Freedberg IM, Eisen AZ, Wolff K, et al, eds. Dermatology in general medicine. New York: McGraw-Hill, 1999.

6. Atherton DJ. The neonate - chronic granulomatous disease. In: Champion RH, Burton JL, Burns DA, Breathnach SM, eds. Textbook of dermatology. Oxford: Blackwell Science, 1998.

7. Lekström-Himes JA, Gallin JI. Immunodeficiency diseases caused by defects in phagocytes. N Engl J Med 2000;343:170-1714.

8. Starke JR, Munoz F. Tuberculosis. In: Behrman RE, Kliegman R, Jenson HB, eds. Nelson textbook of pediatrics. Philadelphia: WB Saunders, 2000.

9. Segal BH, Leto TL, Gallin JI, Malech HL, Holland SM. Genetic, biochemical and clinical features of chronic granulomatous disease. Medicine 2000;79:170-200.

10. Holmes B, Page AR, Good RA. Studies of the metabolic activity of leukocytes from patients with a genetic abnormality of phagocytic function. J Clin Invest 1967;46: 1422-1432.

11. Roos D, Curnutte JT. Chronic granulomatous disease. In: Ochs HD, Smith CIE, Puck JM, eds. Primary immunodeficiency diseases. A molecular and genetic approach. New York: Oxford University Press, 1999:353-374. 
12. Goldblatt D, Thrasher AJ. Chronic granulomatous disease. Clin Exp Immunol 2000;122:1-9.

13. Kobayashi Y, Komazawa Y, Kobayashi M, et al. Presumed BCG infection in a boy with chronic granulomatous disease. Clin Pediatr 1984;23:586-589.

14. Lau YL, Chan GCF, Ha SY, Hui YF, Yuen KY. The role of phagocyte respiratory burst in host defense against
Mycobacterium tuberculosis. Clin Infect Dis 1998;26: 226-227.

15. Kume A, Dinauer MC. Gene therapy for chronic granulomatous disease. J Lab Clin Med 2000;135:122-128.

16. Chowdhury MMU, Anstey A, Matthews CAN. The dermatosis of chronic granulomatous disease. Clin Exp Dermatol 2000;25:190-194. 